\title{
Morphological Aspects of the Inferior Frontal Gyrus and its Relations with Brain Hemispheres and Gender: A Study with in vivo Magnetic Resonance Imaging
}

\author{
${ }^{1}$ Sergio Murilo Georgeto, ${ }^{2}$ Munir Antônio Gariba and ${ }^{3}$ Luiz Roberto Aguiar \\ ${ }^{1}$ Department of Send Message Neurosurgery, Santa Casa Hospital, Brazil \\ ${ }^{2,3}$ Health Technology, Pontifical Catholic University of Paraná, Brazil
}

\section{Article history}

Received: 29-03-2016

Revised: 11-09-2016

Accepted: 09-01-2017

Corresponding Author: Sergio Murilo Georgeto Send Message Neurosurgery Department, Santa Casa Hospital, Brazil Email: georgetosergio@bol.com.br

\begin{abstract}
This study aims to evaluate the reliability of an anatomytopographic location method for the anatomical references that form the inferior frontal gyrus and compare the findings of both studied brain hemisphere as well as the genre. Two different pulse sequences-gradient-echo T1 (T1 GRE) and reversal recovery gradient-echo T1 (T1 IR GRE) were used for such purpose. The sample consisted of 30 healthy individuals with a mean age $25.3 \pm 7.04$ years, 16 women (53\%) and 14 men (46\%). A methodology adapted to identify the limits of the structure forming the inferior frontal gyrus was used. The reliability of the method was evaluated by the level of agreement on intra-examiner and inter-examiner analysis. There was a satisfactory degree of agreement-good, very good or excellent for most references evaluated both on intra-observer and inter-observer analysis. The only exception was the left orbital part on intra -examiner analysis in T1 IR GRE (Kappa test, p>0, 05). No differences were observed between the findings regarding gender. When evaluating the performance between sequences T1 GRE performed better in the independent identification of the structures of the analyzed hemispheres (Kappa test technique Jackniffe, $\mathrm{p}<0.05$ ). The method proved to be reliable for identifying the structures that make up the inferior frontal gyrus. There was no difference between the morphological aspects related to brain hemisphere and gender. Comparing the performance of the sequences, T1 GRE functioned better. However, further studies are needed to confirm such findings.
\end{abstract}

Keywords: Inferior Frontal Gyrus, Anatomy, Magnetic Resonance, Magnetic Resonance Imaging

\section{Introduction}

Neuroanatomical studies are important as they provide a detailed knowledge of specific brain areas (Yasargil et al., 1975). Determining the precise location of anatomical references helps knowledge its funtions as well as the interrelations that they may have (Yaşargil et al., 1988).

There is a current interest in the Inferior Frontal Gyrus morphological aspects (IFG), as they represent a region involved in speech processing and (Almairac et al., 2015) language. IFG is formed by the Orbital Portion (OBP), Triangular Pars (TRP) and Opercular Pars (OPP). TRP is associated with semantic and synthesis functions, whereas OPP is responsible for the articulation of speech (Saur et al., 2008).
The topographical description of such structures that form the IFG originates from classical neuroanatomical studies in cadaver brains. Changes in morphology in previously healthy individuals are described in the literature and are related to both language changes and neuropsychological disorders (Robichon et al., 2000).

There is no standard pulse sequence recommended to analyze the morphological aspects of the cerebral cortex by Magnetic Resonance Imaging (MRI). Recently, both T1 Gradient-Echo (GRE T1) and T2 Gradient-Echo (GRE T2) are used for such purpose (Grossman, 1996).

Advances in MRI equipment allowed IR GRE to be feasible from the economic point of view and could be incorporated in the pulse sequences used in routine tests. This technique provides an enhancement of the cortical grey matter and subcortical white matter, favoring the 
definition of the grooves (sulci) and cortical gyri limits (Meyer et al., 1996).

Literature suggests the use of IR GRE T1 for evaluation of the cortical morphology and recommends its use in the analysis of cortical damage from Multiple Sclerosis (MS) (Geurts et al., 2011). In addition, studies using functional Magnetic Resonance Imaging (fMRI) have demonstrated that there were morphological changes associated with the studied brain hemisphere and gender (Demb et al., 1995).

Therefore, a topographical study using Magnetic Resonance Imaging (MRI) in healthy individuals can assist the knowledge of both peculiarities and similarities regarding IFG anatomy.

This study aimed to propose a topographic location method of the structures that make up the IFG and analyze the degree of confidence. The characteristics of the anatomical references observed were compared to the studied brain hemisphere and gender. Two pulse sequences were chosen to describe the anatomy of GFI and its performance was later observed.

\section{Materials and Methods}

\section{Sample Calculation and Characteristics}

For the sample selection, we used a power test of $80 \%$ and moderate Kappa (kappa > 0.5) in order to test the null hypothesis that there is no agreement between the methods used to identify the structures of the brain lateral surface. From these parameters, it was estimated that the minimum sample would be -25 individuals (Sim and Wright, 2005).

We selected 30 young adults in a non-probabilistic way and by convenience, taking possible losses into account.

\section{Images and Analysis Acquisition}

The selected subjects performed a single skull MRI, T1 GRE and T1 IR GRE images were used. The MRI equipment used is a magneton SYMPHONY $1.5 \mathrm{~T}$ (Siemens, Erlangen, Germany), with 12-channel coil, gradient of $30 \mathrm{mT} / \mathrm{m}$ and a variation rate of $150 \mathrm{mT} / \mathrm{m}$. T1 GRE images were captured with a flip angle of $15^{\circ}$. In the T1 IR GRE images, the reversal time was 350 milliseconds and the parameters of each sequence are shown in Table 1.

The sequence protocols used were based on the manufacturer's recommendations and literature information (11).

The images were initially evaluated by an experienced radiologist to determine their quality and delete possible motion artifacts and pathological findings.

Data were recorded in Digital Imaging and Communications in Medicine system (DICOM), with subsequent images coding and anonymity of volunteers. Next, the images were transferred to a Pro "notebook
MacBook 15 and Osirix MD® v.5.7.1 software 64 bits (Pixmeo SARL, Bernex, Switzerland), which was used to analyze the images of the middle of the orthogonal reconstruction of MPR 2D resources.

T1 GRE and T1 IR GRE files formed 60 image groups, in which the left and right hemispheres were analyzed individually, with a total of 120 groups. Five reviews of random sequences were generated to perform repeatability and reproducibility tests. Each sequence by means of cryptographic pseudo random number developed by Alekcey Colione is available at $<$ Ale.inf.br/encoder $>$.

\section{Identification of the Structures Forming the Inferior Frontal Gyrus}

From 2D images, the anatomy of the brain lateral surface was qualitatively assessed in relation to the identification of key anatomical structures.

A method for topographic location based on literature data was developed (Naidich et al., 1995). The step by step method was divided into five stages according to the following description (Fig. 1):

Step 1: To determine the image of the Lateral Fissure (LF) at its widest extension: Using the sagittal plane, we seek to set the LF at its widest. To identify the limits of the following references, FL image shift may be necessary both laterally and medially to its widest extension original plan.

Step 2: Location of the inferior frontal gyrus (IFG)-The IFG is located superiorly to the LF at the frontoparietal operculum and is partially enclosed in its dorsal limit by Superior Frontal Sulcus (SFS) and its ventral limit by the rostral portion of the LF. The Horizontal Anterior Branch (AHB) and Ascending Anterior Branch (AAB) arise from LF in its infero-rostral-caudal part dividing GFI in three parts: The opercular part (OPP), the Triangular Portion (TRP) and Orbital Part (OBP).

Step 3: The opercular portion (OPP)-in the sagittal plane may have the shape of a single or double spin. Its dorsal limit is the union of the distal inferior frontal sulcus portion (SFI) with the ventral branch of the lower end of the pre-central sulcus. Its ventral limit is formed by the ascending branch of the lateral horizontal fissure. It has as cranial limit the RAA and as caudal limit the lower end of the pre-central sulcus.

Step 4: The Triangular Part (TRP)-in the sagittal plane. It forms an inverted triangle whose upper limit is the lower front sulcus, which extends to the average portion of the base of the triangle where, a Triangular Sulcus (TS) or capitis notch are sometimes found. The continuity of TS is the Inferior Frontal Sulcus (IFS). The lower limit of 
TRP is the ascending branch of the lateral fissure, while the Previous Horizontal Branch (AHB) is located rostrally and the ascending Anterior Branch $(\mathrm{AB})$ is located caudally. The location of these two references is essential for the delimitation of the TRP. Sometimes the presence of a groove in the rear portion of the TRP is observed and it is called Diagonal Sulcus (DS). This groove/sulcus-when present-is found prior to the ascending Sub-Central Sulcus (SSCA).

Step 5: The Orbital Part (OBP)-it has as dorsal limit the Lateral Frontal-Marginal Sulcus (LFMS). On the ventral portion of the OBP is the previous ascending limb (CEOS) of the FL.

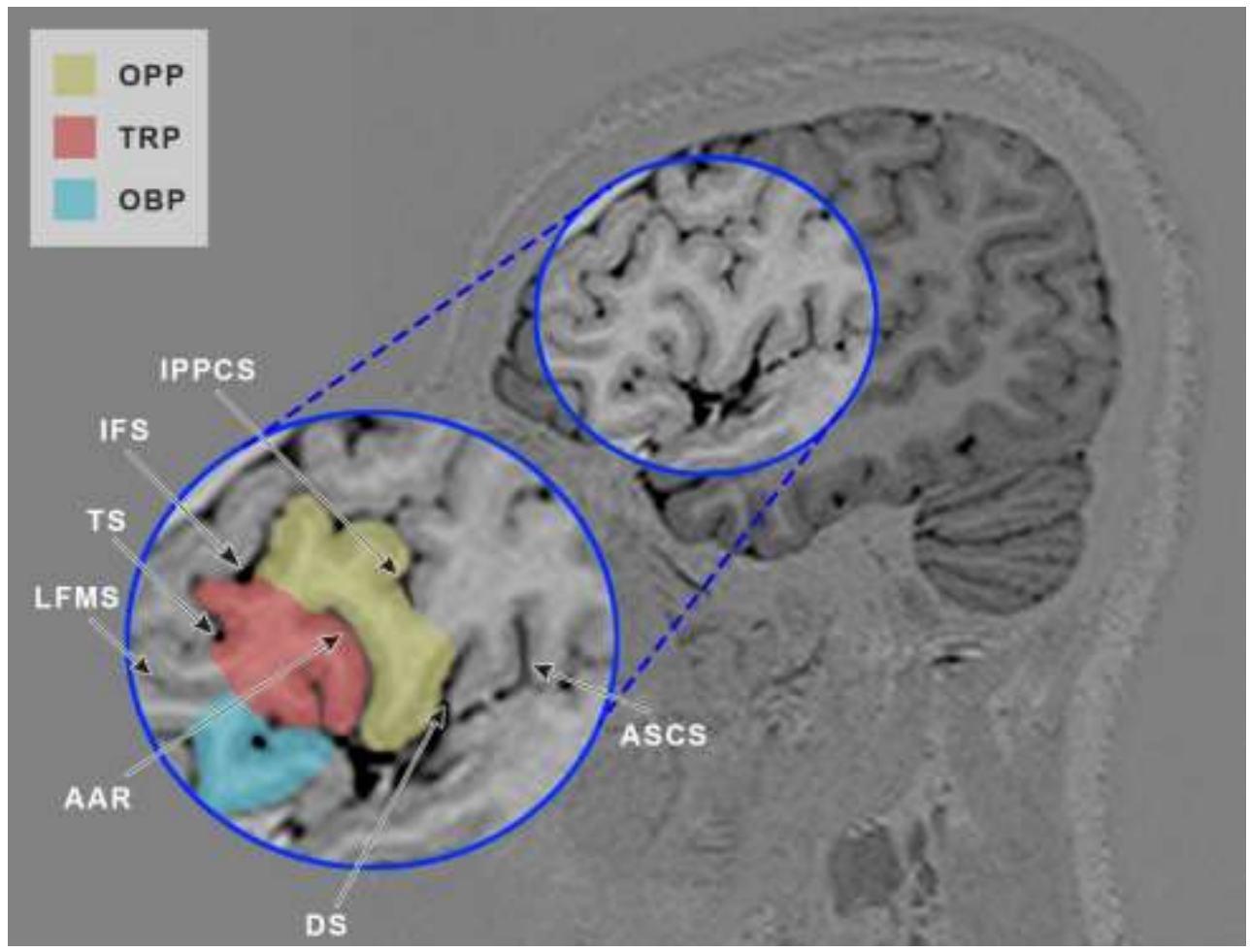

Fig. 1. Illustration of the above steps

Table 1. Specifications of MRI capturing parameters on both sequences (T1 GRE and T1 IR)

\begin{tabular}{lll}
\hline & T1 GRE & T1 IR GRE \\
\hline Capturing & Sagittal volumetric Gradient-echo & Coronal volumetric \\
Matrix & $256 \times 256$ & $256 \times 256$ \\
Voxel & Isotropic $(1 \times 1 \times 1 \mathrm{~mm})$ & Isotropic $(1 \times 1 \times 1 \mathrm{~mm})$ \\
Parameters & TR 1910 & TR 400 \\
& TE 3.09 & TE 373 \\
FOV & $256 \mathrm{~mm}$ & $260 \mathrm{~mm}$ \\
Cutthickness & $1 \mathrm{~mm}$ & $1 \mathrm{~mm}$ \\
Interval between cuts & Zero & Zero \\
Flipangle & $15^{\circ}$ & - \\
Inversion time & - & $350 \mathrm{milli} \mathrm{sec}$ \\
\hline
\end{tabular}

Table 2. Concordance standard with prevalence-adjusted and bias-adjusted Kappa, according to the criteria pointed out by Byrt et al. (1993; Duvernoy, 1999)

\begin{tabular}{ll}
\hline $0.93-1.00$ & Excellent \\
$0.81-0.92$ & Very good \\
$0.61-0.80$ & Good \\
$0.41-0.60$ & Satisfactory \\
$0.21-0.40$ & Weak \\
$0.01-0.20$ & Poor \\
0.00 or lower & Without concordance \\
\hline
\end{tabular}




\section{Reliability Analysis Method (Repeatability and Reproducibility)}

The anatomical references were classified as easily identifiable (2), on a clear identification mode (1) and unidentifiable $(0)$.

The researcher (SMG) has made three observations guided by the proposed method with a 10-day interval each between each test of repeatability.

In order to confirm the reproducibility of the method, two neurosurgeons have been invited. They received a manual with instructions on how to use the proposed method. The comparison between the evaluation of the two neurosurgeons was added to the first investigator assessment, resulting in a total of three to confirm the reproducibility of the method.

The standards agreement was based on the classification with prevalence and bias adjustment (Kappa) according to the data presented in Table 2 (Byrt et al., 1993).

\section{Statistical Analysis}

The results obtained in this study were described as absolute $(\mathrm{N})$ and relative frequency percentage $(\mathrm{P})$.

To demonstrate the results-method in relation to the pulse sequences used (T1 GRE and T1 IR GRE), as well as to the comparison of the right and left brain hemispheres and gender. As for the display mode, the anatomical structures that form GFI were divided into two distinct classes, as observation:

The results were unclear about its identity were added to those unidentifiable versus the easily identifiable findings. Data analysis was performed using Fisher's exact test, with $\mathrm{p}, 0,05$ indicating statistical significance.

Kappa coefficient was used to analyze the correlation between the three evaluations of the same researcher (agreement) and between assessments of different examiners (interobservers); $\mathrm{p}$ values $<0.05$ indicate statistical significance. Data were analyzed using computer software Stata v.13.1.

To verify the evaluation effectiveness T1 GRE versus T1 IR GRE, Kappa indices were compared in different structures of inferior frontal gyrus, with the Jackknife technique in computer software 3.1 Epidat.

\section{Results}

\section{Sample Characteristics}

Thirty young adults (average age $25.3 \pm 7.04$ years) participated in this study, 16 women $(53.3 \%)$ and 14 men (46.7\%). Six of the 16 women were left-handed (37.5\%) and the other 10 were right-handed $(62.5 \%)$; seven of the 14 men $(50 \%)$ were left-handed, while the other seven $(50 \%)$ were right-handed. They had no history of neurological or psychiatric disorders. For manual dexterity test, the Inventory Edinburgh Manual was used.

\section{Method Reliability Analysis (Intra and Inter- Examiner Reproducibility) to Determine the Inferior Frontal Gyrus Structures}

We observed the degree of intraobserver agreement ranging from satisfactory, good, very good or excellent for the right structures analyzed by the technique T1 IR GRE (Table 4).

Table 3. Intra-observer concordance for featuring the brain lateral surface structures, according to the T1 IR GRE and T1 GRE methods, on right and left sides

\begin{tabular}{|c|c|c|c|c|c|c|}
\hline \multirow[b]{2}{*}{ Variable } & \multicolumn{4}{|c|}{ Intraobserver concordance } & \multirow[b]{2}{*}{ KAPPA } & \multirow[b]{2}{*}{$\mathrm{p}$ value } \\
\hline & $\begin{array}{l}\text { Concordance } \\
\text { non-identifiable }\end{array}$ & $\begin{array}{l}\text { Concordance } \\
\text { unclear }\end{array}$ & $\begin{array}{l}\text { Concordance } \\
\text { easily identifiable }\end{array}$ & Non-concordance & & \\
\hline \multicolumn{7}{|l|}{ T1 IR GRE-Right Side } \\
\hline Inferior frontal gyrus & $0(0)$ & $1(3,3)$ & $28(93,3)$ & $1(3,3)$ & 0,79 & $<0,001$ \\
\hline Orbital Part & $0(0)$ & $4(13,3)$ & $23(76,7)$ & $3(10)$ & 0,79 & $<0,001$ \\
\hline Triangular Part & $0(0)$ & $7(23,3)$ & $22(73,3)$ & $1(3,3)$ & 0,94 & $<0,001$ \\
\hline Opercular Part & $0(0)$ & $4(13,3)$ & $19(63,3)$ & $7(23,3)$ & 0,59 & $<0,001$ \\
\hline \multicolumn{7}{|l|}{ T1 IR GRE-Left Side } \\
\hline Inferior frontal gyrus & $0(0)$ & $0(0)$ & $28(93,3)$ & $2(6,7)$ & - & - \\
\hline Orbital part & $0(0)$ & $0(0)$ & $28(93,3)$ & $2(6,7)$ & $0,31 * * *$ & 0,002 \\
\hline Triangular part & $0(0)$ & $2(6,7)$ & $26(86,7)$ & $2(6,7)$ & 0,78 & $<0,001$ \\
\hline Opercular part & $0(0)$ & $4(13,3)$ & $23(76,7)$ & $3(10)$ & 0,78 & $<0,001$ \\
\hline \multicolumn{7}{|l|}{ T1 IR GRE-Right Side } \\
\hline Inferior Frontal Gyrus & $0(0)$ & $1(3,3)$ & $28(93,3)$ & $1(3,3)$ & 0,79 & $<0,001$ \\
\hline Orbital Part & $0(0)$ & $5(16,7)$ & $23(76,7)$ & $2(6,7)$ & 0,87 & $<0,001$ \\
\hline Triangular Part & $0(0)$ & $7(23,3)$ & $22(73,3)$ & $1(3,3)$ & 0,94 & $<0,001$ \\
\hline Opercular Part & $0(0)$ & $6(20)$ & $20(66,7)$ & $4(13,3)$ & 0,78 & $<0,001$ \\
\hline \multicolumn{7}{|l|}{ T1 IR GRE-Left Side } \\
\hline Inferior Frontal Gyrus & $0(0)$ & $0(0)$ & $28(93,3)$ & $2(6,7)$ & - & - \\
\hline Orbital Part & $0(0)$ & $0(0)$ & $28(93,3)$ & $2(6,7)$ & - & - \\
\hline Triangular Part & $0(0)$ & $2(6,7)$ & $26(86,7)$ & $2(6,7)$ & 0,77 & $<0,001$ \\
\hline Opercular Part & $0(0)$ & $4(13,3)$ & $22(73,3)$ & $4(13,3)$ & 0,72 & $<0,001$ \\
\hline
\end{tabular}

Concordance*, poor** and weak*** 
Table 4.Inter-observer concordance for featuring the brain lateral surface structures, according to the T1 IR GRE and T1 GRE methods, on right and left sides

\begin{tabular}{|c|c|c|c|c|c|c|}
\hline \multirow[b]{2}{*}{ Variable } & \multicolumn{6}{|c|}{ Intraobserver concordance } \\
\hline & $\begin{array}{l}\text { Concordance } \\
\text { non-identifiable }\end{array}$ & $\begin{array}{l}\text { Concordance } \\
\text { unclear }\end{array}$ & $\begin{array}{l}\text { Concordance } \\
\text { easily identifiable }\end{array}$ & Non-concordance & KAPPA & $\mathrm{p}$ value \\
\hline \multicolumn{7}{|l|}{ T1 IR GRE-Right Side } \\
\hline Inferior Frontal Gyrus & $0(0)$ & $0(0)$ & $28(93,3)$ & $2(6,7)$ & - & - \\
\hline Orbital Part & $0(0)$ & $5(16,7)$ & $23(76,7)$ & $2(6,7)$ & 0,86 & $<0,001$ \\
\hline Triangular Part & $0(0)$ & $7(23,3)$ & $22(73,3)$ & $1(3,3)$ & 0,94 & $<0,001$ \\
\hline \multirow{2}{*}{\multicolumn{7}{|c|}{ T1 IR GRE-Left Side }} \\
\hline & & & & & & \\
\hline Inferior Frontal Gyrus & $0(0)$ & $0(0)$ & $30(100)$ & $0(0)$ & - & - \\
\hline Orbital Part & $0(0)$ & $0(0)$ & $29(96,7)$ & $1(3,3)$ & 0,49 & $<0,001$ \\
\hline Triangular Part & $0(0)$ & $3(10)$ & $26(86,7)$ & $1(3,3)$ & 0,89 & $<0,001$ \\
\hline Opercular Part & $0(0)$ & $3(10)$ & $23(76,7)$ & $4(13,3)$ & 0,67 & $<0,001$ \\
\hline \multicolumn{7}{|l|}{ T1 GRE-Right Side } \\
\hline Inferior Frontal Gyrus & $0(0)$ & $0(0)$ & $28(93,3)$ & $2(6,7)$ & - & - \\
\hline Orbital Part & $0(0)$ & $6(20)$ & $23(76,7)$ & $1(3,3)$ & 0,93 & $<0,001$ \\
\hline Triangular Part & $0(0)$ & $8(26,7)$ & $22(73,3)$ & $0(0)$ & 1 & $<0,001$ \\
\hline Opercular Part & $0(0)$ & $3(10)$ & $21(70)$ & $6(20)$ & 0,52 & $<0,001$ \\
\hline \multicolumn{7}{|l|}{ T1 GRE-Left Side } \\
\hline Inferior Frontal Gyrus & $0(0)$ & $0(0)$ & $30(100)$ & $0(0)$ & - & - \\
\hline Orbital Part & $0(0)$ & $0(0)$ & $29(96,7)$ & $1(3,3)$ & - & - \\
\hline Triangular Part & $0(0)$ & $3(10)$ & $26(86,7)$ & $1(3,3)$ & 0,49 & $<0,001$ \\
\hline Opercular Part & $0(0)$ & $4(13,3)$ & $22(73,3)$ & $4(13,3)$ & 0,87 & $<0,001$ \\
\hline
\end{tabular}

Table 5. Comparison between genders on the identification of inferior frontal gyrus structures

\begin{tabular}{|c|c|c|c|c|}
\hline \multirow[b]{2}{*}{ Variables } & \multirow[b]{2}{*}{ Classification } & \multicolumn{2}{|l|}{ Gender } & \multirow[b]{2}{*}{$\mathrm{p}$} \\
\hline & & Female & Male & \\
\hline \multicolumn{5}{|l|}{ Right Side-T1 IR } \\
\hline \multirow[t]{2}{*}{ Inferior frontal gyrus } & Unclear or non-identified & $1(6,3)$ & $1(7,1)$ & \\
\hline & Easily identified & $15(93,8)$ & $13(92,9)$ & 1 \\
\hline \multirow[t]{2}{*}{ Orbital part } & Unclear or non-identified & $3(18,8)$ & $2(14,3)$ & \\
\hline & Easily identified & $13(81,3)$ & $12(85,7)$ & 1 \\
\hline \multirow[t]{2}{*}{ Triangular part } & Unclear or non-identified & $4(25)$ & $4(28,6)$ & \\
\hline & Easily identified & $12(75)$ & $10(71,4)$ & 1 \\
\hline \multirow{2}{*}{ Opercular part } & Unclear or non-identified & $3(18,8)$ & $8(57,1)$ & \\
\hline & Easily identified & $13(81,3)$ & $6(42,9)$ & 0,057 \\
\hline \multicolumn{5}{|l|}{ Left Side-T1 IR } \\
\hline \multirow[t]{2}{*}{ Inferior frontal gyrus } & Unclear or non-identified & $1(6,3)$ & $1(7,1)$ & \\
\hline & Easily identified & $15(93,8)$ & $13(92,9)$ & 1 \\
\hline \multirow[t]{2}{*}{ Orbital part } & Unclear or non-identified & $0(0)$ & $1(7,1)$ & \\
\hline & Easily identified & $16(100)$ & $13(92,9)$ & 0,467 \\
\hline \multirow[t]{2}{*}{ Triangular part } & Unclear or non-identified & $1(6,3)$ & $2(14,3)$ & \\
\hline & Easily identified & $15(93,8)$ & $12(85,7)$ & 0,586 \\
\hline \multirow[t]{2}{*}{ Opercular part } & Unclear or non-identified & $3(18,8)$ & $3(21,4)$ & \\
\hline & Easily identified & $13(81,3)$ & $11(78,6)$ & 1 \\
\hline \multicolumn{5}{|l|}{ Right Side-T1 } \\
\hline \multirow[t]{2}{*}{ Inferior frontal gyrus } & Unclear or non-identified & $1(6,3)$ & $1(7,1)$ & \\
\hline & Easily identified & $15(93,8)$ & $13(92,9)$ & 1 \\
\hline \multirow[t]{2}{*}{ Orbital part } & Unclear or non-identified & $3(18,8)$ & $3(21,4)$ & \\
\hline & Easily identified & $13(81,3)$ & $11(78,6)$ & 1 \\
\hline \multirow[t]{2}{*}{ Triangular part } & Unclear or non-identified & $4(25)$ & $4(28,6)$ & \\
\hline & Easily identified & $12(75)$ & $10(71,4)$ & 1 \\
\hline \multirow[t]{2}{*}{ Opercular part } & Unclear or non-identified & $2(12,5)$ & $7(50)$ & \\
\hline & Easily identified & $14(87,5)$ & $7(50)$ & 0,056 \\
\hline \multicolumn{5}{|l|}{ Left Side-T1 } \\
\hline \multirow{2}{*}{ Inferior frontal gyrus } & Unclear or non-identified & $1(6,3)$ & $1(7,1)$ & \\
\hline & Easily identified & $15(93,8)$ & $13(92,9)$ & 1 \\
\hline \multirow[t]{2}{*}{ Orbital part } & Unclear or non-identified & $0(0)$ & $1(7,1)$ & \\
\hline & Easily identified & $16(100)$ & $13(92,9)$ & 0,467 \\
\hline \multirow[t]{2}{*}{ Triangular part } & Unclear or non-identified & $1(6,3)$ & $2(14,3)$ & \\
\hline & Easily identified & $15(93,8)$ & $12(85,7)$ & 0,586 \\
\hline \multirow[t]{2}{*}{ Opercular part } & Unclear or non-identified & $3(18,8)$ & $3(21,4)$ & \\
\hline & Easily identified & $13(81,3)$ & $11(78,6)$ & 1 \\
\hline
\end{tabular}


Nevertheless, there was a satisfactory degree of concordance, very good or excellent to the left side through the T1 GRE method, except the left orbital (Table 3).

Regarding the right hemisphere structures identification T1 GRE, there was a level of agreement ranging from satisfactory to good, very good or excellent degree for all analyzed structures. Moreover, the identification of the references to the left hemisphere T1 GRE, the degree of concordance was found satisfactory, good, very good or excellent for the analysed structures (Table 3).

This study examined the degree of inter-observer agreement ranging from satisfactory, good, very good and excellent for the analyzed references analyzed in left and right sides by T1 GRE and T1 IR GRE methods (Table 4).

Table 4- Inter-observer concordance for featuring the brain lateral surface structures, according to the T1 IR GRE and T1 GRE methods, on right and left sides.

\section{Comparison between Genders of the Inferior Frontal Gyrus Structures}

There were no anatomo-topographic differences in relation to gender in GFI (Table 5).

\section{Comparison of Methods to Identify the Structures of Inferior Frontal Gyrus}

To check the effectiveness of T1 GRE against T1 IR GRE, a comparison of Kappa concordance degree to different GFI structures was performed. The Jackknife technique was used and showed a better performance of the T1 GRE technique to identify the morphology of GFI structures, regardless of the hemisphere analyzed.

\section{Discussion}

The IFG is a complex structure, with morphological variations that tend to follow specific patterns. The anatomy atlases by MRI images show the structures that comprise the IFG in details, but they do not indicate a method to identify anatomical references (Duvernoy, 1999; Damasio, 2005).

There are variations of classic patterns described in healthy subjects (Geschwind and Galaburda, 1985) and some of these morphological changes are related to language disorders (Leonard and Eckert, 2008). Furthermore, cerebral asymmetry patterns may have different symptoms according to the affected hemisphere (Knaus et al., 2006).

Structural changes may be due to inaccurate anatomical descriptions. Regarding IFG, these differences may be explained by three reasons: difficulty of defining its back-orbital limit; Discrepancies between references that are used, such as back-orbital margin; similar names assigned to different structures.

The back-orbital rim is formed by the pretriangular sulcus and by the lateral portion of the frontal-marginal sulcus. They are tertiary sulci, however they present a frequency that allows them to be used as anatomical references (Petrides and Pandya, 2012). The presence of these two sulci was observed in $28 \%$ of left hemispheres in $12 \%$ of right hemispheres (Ono et al., 1990).

In literature there are different names for the same anatomical structure. This is the case of FrontalMarginal Sulcus (LPFMS), which is also called Lateral Orbital Sulcus (LOS) (Duvernoy et al., 2013). Some authors consider them as being a complex of the $\mathrm{H}$ shaped notches on the bottom surface of the frontal lobe (Bailey and Bonin, 1951). However, there are other reports that describe that the LPFMS is situated laterally and dorsally to the LOS (Petrides and Sulci, 2012).

A flaw in the method described in this work is related to the pattern of morphological changes used as a reference. There are reports of topographical changes in healthy individuals (Knaus et al., 2006) and these may be related to language disorders (Leonard and Eckert, 2008).

The combination of images obtained through different sequences of MRI is one of the reasons for neuroanatomical studies of brain surface (Li et al., 2013) and for neurosurgical planning (Bai et al., 2015).

The T1 GRE technique proved superior in identifying structures, regardless of the analyzed brain hemisphere, of the sample investigated. The description of a reliable method (repeatable and reproducible) can be useful to practitioners involved in both the diagnosis and treatment of diseases affecting this region.

\section{Conclusion}

This study demonstrated a reliable method in analyzing the repeatability and reproducibility for both T1 GRE and T1 IR GRE techniques.

There were no differences between the neuroanatomical findings related to the studied brain hemisphere and gender.

The technique T1 GRE presented better performance compared to T1 IR GRE for location of structures that make up IFG in this sample. However, further studies are needed to confirm the results.

\section{Acknowledgement}

The authors gratefully acknowledge the technical assistance of Mr. Alekcey Gavioli Colione. The authors are especially indebted to Dr. Heraldo Mello Neto ( XLeme Clinic) for the MR images.

\section{Funding Information}

This study wasn't supported by any institution.

\section{Author's Contributions}

All authors equally contributed in this work. 


\section{Conflict of Interest}

The authors declare no conflict of interest regarding the publication of this article.

\section{References}

Almairac, F., G. Hebert, G. Moritz-Gasser, N.M. Champfleur and H. Duffau, 2015. The left inferior fronto-occipital fasciculus subserves language semantics: A multilevel lesion study. Brain Structure Funct., 220: 1983-1995.

DOI: $10.1007 / \mathrm{s} 00429-014-0773-1$

Bai, S., B. Xu, S. Wei, J. Geng and D. Wu, 2015. Intraoperative high-field magnetic resonance imaging combined with functional neuronavigation in resection of low-grade temporal lobe tumors. World J. Surgical Oncol., 13: 1-7.

DOI: $10.1186 / \mathrm{s} 12957-015-0690-7$

Bailey, P. and G.V. Bonin, 1951. Fissures of the Brain. In: The Isocortex of Man, Bailey, P. and G.V. Bonin (Ed.), University of Illinois Press, Urbana, pp: 21-61.

Byrt, T., J. Bishop and J.B. Carlin, 1993. Bias, prevalence and kappa. J. Clin. Epidemiol., 46: 423-429. PMID: 8501467

Damasio, H., 2005. Exterior Description of Normal Dolicocephalic Brain. In: Human Brain Anatomy in Computerized Images. Damasio, H. (Ed.), Oxford University Press, New York, ISBN-10: 0198037058, pp: 1-6.

Demb, J.B., J.E. Desmond, A.D. Wagner, C.J. Vaidya and G.H. Glover et al., 1995. Semantic encoding and retrieval in the left inferior prefrontal cortex: A functional MRI study of task difficulty and process specificity. J. Neurosci., 15: 5870-5878. PMID: 7666172

Duvernoy, H., F. Cattin and P. Risold, 2013. Sectional Anatomy and Magnetic Resonance Imaging. In: The Human Hippocampus: Functional Anatomy, Vascularization and Serial Sections with MRI, Duvernoy, H.M., F. Cattin, P.Y. Risold, (Eds.), Springer Science \& Business Media, Berlin, ISBN-10: 3642336035, pp: 127-213.

Duvernoy, H.M., 1999. Sagital Sections. In: The Human Brain: Surface, Three-Dimensional Sectional Anatomy with MRI and Blood Supply, Duvernoy, H.M. (Ed.), Springer Science \& Business Media, Wien, ISBN-10: 3211831584, pp: 252-309.

Geschwind, N. and A.M. Galaburda, 1985. Cerebral lateralization. Biological mechanisms, associations and pathology: I. A hypothesis and a program for research. Arch. Neurol., 42: 428-459. PMID: 3994562

Geurts, J., S. Roosendaal, M. Calabrese, O. Ciccarelli and F. Agosta et al., 2011. Consensus recommendations for MS cortical lesion scoring using double inversion recovery MRI. Neurology, 76: 418-424. DOI: 10.1212/WNL.0b013e31820a0cc4
Grossman, C.B., 1996. Physical Principles of Computed Tomography and Magnetic Resonance Imaging, In: Magnetic Resonance Imaging and Computed Tomography of the Head and Spine, Wilkins, W.A. (Ed.), Williams and Wilkins, Baltimore, ISBN-10: 0683037692, pp: 10-54.

Knaus, T.A., A.M. Bollich, D.M. Corey, L.C. Lemen and A.L. Foundas, 2006. Variability in perisylvian brain anatomy in healthy adults. Brain Lang., 97: 219-232. PMID: 16300824

Leonard, C.M. and M.A. Eckert, 2008. Asymmetry and dyslexia. Develop. Neuropsychol., 33: 663-681. DOI: $10.1080 / 87565640802418597$

Li, A.W., P. Nopoulos and V.A. Magnotta, 2013. Automated parcellation of the brain surface generated from magnetic resonance images. Front. Neuroinformat., 7: 1-11. PMID: 24155718

Meyer, J.R., S. Roychowdhury, E.J. Russell, C. Callahan and D. Gitelman et al., 1996. Location of the central sulcus via cortical thickness of the precentral and postcentral gyri on MR. Am. J. Neuroradiol., 17: 1699-1706. PMID: 8896626

Naidich, T.P., A.G. Valavanis and S. Kubik, 1995. Anatomic relationships along the low-middle convexity: Part I--Normal specimens and magnetic resonance imaging. Neurosurgery, 36: 517-532. PMID: 7753352.

Ono, M., S. Kubik and C.D. Abernathey, 1990. Introduction, Nomenclature, Classification. In: Atlas of the Cerebral Sulci, Ono, M., S. Kubik and C.D. Abernathey (Ed.), Thieme, Stuttgart, ISBN-10: 0865773629, pp: 2-149.

Petrides, M. and A. Sulci, 2012. Gyri of Cerebral Cortex. In: The Human Cerebral Cortex: An MRI Atlas of Sulci and Gyri in Stereotaxi Space, Petrides, M. (Ed.), Elsevier, London, pp: 25-53.

Petrides, M. and D. Pandya, 2012. The Frontal Cortex. In: The Human Nervous System, Mai, J.K. and G. Paxinos (Ed.), Elsevier Academic Press, Academic Press, ISBN-10: 0123742366, pp: 988-995.

Robichon, F., O. Levrier, P. Farnarier and M. Habib, 2000. Developmental dyslexia: Atypical cortical asymmetries and functional significance. Eur. J. Neurol., 7: 35-46. DOI: 10.1046/j.1468-1331.2000.00020.x

Saur, D., B.W. Kreher, S. Schnell, D. Kümmerer and P. Kellmeyer et al., 2008. Ventral and dorsal pathways for language. Proc. National Acad. Sci., 105: 18035-18040. DOI: 10.1073/pnas.0805234105

Sim, J. and C.C. Wright, 2005. The kappa statistic in reliability studies: Use, interpretation and sample size requirements. Phys. Therapy, 85: 257-268. PMID: 15733050

Yaşargil, M., G. Cravens and P. Roth, 1988. Surgical approaches to "inaccessible" brain tumors. Clin. Neurosurgery, 34: 42-110. PMID: 3288405

Yasargil, M.G., K. Kasdaglis and K.K. Jain, 1975. The operative approach to aneurysms of the anterior communicating artery. Adv. Tech. Standards Neurosurgery, 2: 114-170. 